\title{
Using 2D and 3D strain to detect myocardial damage during anthracycline chemotherapy in breast cancer patients
}

\author{
Fang-Yi Tao ${ }^{1}$, Yin-Li Luo ${ }^{1}$, Jing-Hong Xu ${ }^{1}$, Shu-Ting Zhu ${ }^{1}$, Bin-Bin Shi ${ }^{1}$, Zeng-Hui \\ Liang $^{1}$, and Bin Chen ${ }^{1}$ \\ ${ }^{1}$ The First Affiliated Hospital of Wenzhou Medical University
}

October 28, 2020

\begin{abstract}
Both two-dimensional (2D) and three-dimensional (3D) echocardiography can be used in the early diagnosis of myocardial toxicity in patients with anthracycline chemotherapy. However, there are few studies on the detection of early myocardial damage in specific regions. This study compared the role and significance of $2 \mathrm{D}$ strain and $3 \mathrm{D}$ strain in discovering early segmental dysfunction. We prospectively studied 56 breast cancer patients who received anthracycline therapy. The mean age of patients was $47.6 \pm 8.1$ years. They all received $4-6$ cycles of chemotherapy. Before chemotherapy and after every two cycles, patients underwent standard echo, 2D and 3D speckle-tracking echocardiography (STE) and real-time three-dimensional echocardiography (RT-3DE). Compared with the baseline value (T0), 3D GLS was significantly reduced after two cycles (T2) $(\mathrm{P}<0.05)$, after four cycles (T4) 3D GCS was significantly reduced $(\mathrm{P}<0.05)$, after six cycles $(\mathrm{T} 6)$ 2D derived EF, 2D GLS, E/e' ratio, Fractional shortening (FS), 3D derived EF, 3D GAS, GRS were considerably decreased $(\mathrm{P}<0.05)$. The area supplying blood from the anterior descending branch of the left coronary artery was the most susceptible to chemotherapy, and 3D GLS was found earlier than 2D GLS which was decreased considerably after two cycles $(\mathrm{P}<0.05)$. In anthracycline chemotherapy patients, some regions of early myocardial dysfunction may be more easily involved, through the evaluation of $2 \mathrm{D}$ and $3 \mathrm{D}$ echocardiography, 3D speckle tracking echocardiography may be more able to identify the involved segments of myocardium early. Moreover, the apical segments of the left ventricle seem to be more susceptible to cardiotoxicity.
\end{abstract}

\section{Introduction}

The survival rate of cancer patients has been significantly improved in recent decades. Because of the development of early diagnosis techniques, improved in the level of treatment and the implementation of multidisciplinary treatment of cancer, and advances in adjuvant chemotherapy and radiotherapy[1]. Although with the development of anti-tumor drug therapy towards the direction of individual and targeted therapy, its toxic and side effects should not be ignored. Research has shown that about half of cancer deaths are caused by non-cancer causes, and cardiovascular disease plays an important role[2].

Anthracyclines are secondary metabolites produced by Streptomyces and are one of the most effective anticancer drugs at present. It mainly includes daunorubicin, Adriamycin, epirubicin, pirarubicin, mitoxantrone and so on. Although the field of cancer treatment has changed dramatically in recent decades, anthracyclines remain the cornerstone of modern chemotherapy regimens for many cancers[3, 4]. However, the most severe adverse reaction of anthracyclines is the cardiotoxicity caused by anthracyclines. Numerous trials have indicated that most of the cardiotoxicity caused by anthracyclines are persistent and non-reversing, especially the first use of anthracyclines easily cause cardiac damage. Therefore, early detection of cardiac insufficiency can implement cardiac protection strategies before late and potentially irreversible changes in cardiac function $[5,6]$.

Echocardiography is a well-known non-invasive technique for assessment of cardiac systolic and diastolic 
function, and it is the most commonly used method for early identification and evaluation of cardiotoxicity caused by antineoplastic drugs. Left-ventricular ejection fraction (LVEF) is the most frequently used index to evaluate left ventricular systolic function by conventional echocardiography, and the decrease of LVEF is related to heart failure. However, its sensitivity to early identification of cardiotoxicity is still controversial[7]. The cardiotoxicity induced by anthracyclines was mostly positively correlated with the dose, and in China the dose of anthracycline in the treatment of breast cancer is relatively low. Therefore, more sensitive monitoring methods are needed to monitor the cardiotoxicity associated with low-dose anthracyclines. In recent years, with the development of echocardiography technology, 2D and 3D speckle-tracking echocardiographic parameters have been proved to be able to detect preclinical abnormalities reliably at an early stage [8-10]. Standard echocardiography can detect abnormalities in the presence of obvious clinical symptoms. In the preclinical state, strain imaging can make up for its deficiency and is of great help in early diagnosis and define prognosis.[11].

However, because of the different distribution of myocardial fibres, some areas of the myocardium may be more prone to dysfunction[12]. This study aimed to compare the role and significance of 2D strain and 3D strain in the detection of early segmental dysfunction, and to explore which is more meaningful, including 3D global strain, longitudinal strain, radial strain and circumferential strain.

\section{Materials and methods}

\section{Study population}

This prospective observational study screened more than 100 breast cancer patients from April 2019 to May 2020 and plans to receive anthracycline chemotherapy at the first affiliated Hospital of Wenzhou Medical University. A detailed questionnaire was gathered, including the presence of clinical history, electrocardiogram, an echocardiogram, and blood sampling, at the recruitment. This study protocol was approved by the Ethics Committee of the First Affiliated Hospital of Wen Zhou Medical University.

Inclusion criteria were: at least 18 years of age; have not received chemotherapy and (or) radiotherapy before; electrocardiogram (ECG), kidney and liver function, blood routine tests were standard; breast cancer was diagnosed by histopathology in our hospital, and at least four cycles of chemotherapy were completed in our hospital.

Exclusion criteria were: pregnant or nursing women; metastatic breast cancer; congenital heart disease; valvular disease; severe arrhythmia; standard echo images of inadequate quality and echocardiographic $\mathrm{EF}<50 \%$ before chemotherapy.

The primary end-point was patients with significant LVEF decline without congestive heart failure symptoms or mild symptoms (NYHA heart function class I), LVEF decreased by at least $10 \%$ and LVEF $<53 \%$; Patients with symptoms of congestive heart failure (NYHA heart function grade II, III or IV grade), LVEF decreased by at least $5 \%$ and LVEF $<53 \%$; GLS decreased by more than $15 \%$ compared with the baseline value before chemotherapy according to the ASE/EACVI Expert Consensus[13-15].

Finally, 56 patients met the criteria for enrollment. All patients will be informed of the study aim and design and signed informed consent. Each patient was then studied before chemotherapy, after the first chemotherapy, and completion of anthracyclines therapy. The ATN dose, heart rate, height and weight of the patients were recorded at the same time of each study.

\section{Echocardiographic methods}

All transthoracic echocardiographic data were performed by a GE Vivid E95 ultrasound machine (GE Healthcare, Oslo, Norway) with cardiac sector probe M5S and 4V (1.0-1.5 Hz), and using Echopac workstation (Version:201) for offline analyses. Connect the ECG to record the moving image of at least five cardiac cycles (frame rate $>40 \mathrm{fps}$ ), to ensure the image is clear (endocardium) and complete (including epicardium), and adjust the frame rate to $40 \%$ of the patient's heart rate. All echocardiograms of each patient were obtained by the same ultrasound machine. 


\section{Standard ultrasoundexamination.}

Along the parasternal LV long-axis view measured the LV end-systolic diameter (LVIDS), LV end-diastolic diameter (LVIDD), LV posterior wall thickness (LVPW), end-systolic volume (ESV), end-diastolic volume (EDV). LVEF as indicators to assess left ventricular systolic function was calculated by the modified Simpson biplane method, with images recoded from apical two-chamber and apical four-chamber views. Fractional shortening (FS) was derived from M-mode imaging. In the apical four-chamber section, the mean value of early diastolic peak velocity (e') of septal and lateral mitral annulus was computed by tissue Doppler and pulsed Doppler imaging. The E/e' ratio was calculated by the ratio of the peak early transmission velocity (E) to the average e'. Then FS and E/e' ratio could be used to evaluate left ventricular diastolic function [16]. Moreover, we used M-mode and tissue Doppler imaging echocardiography methods to measure isovolumic relaxation time (IRT), isovolumic contraction time (ICT) and ejection time (ET), and then Tei index((IRT+ICT)/ET) was computed automatically by the ultrasonic system as a reliable estimate of left ventricular global function[17]. These data were expressed as averages of three consecutive cycles.

\section{RT-3DE examination}

The $4 \mathrm{~V}$ probe captured 3D full volume images in wide-angle mode when holding the breath at the end of exhalation. Five fan-shaped images were obtained through five consecutive cardiac cycles, and the volume ratio of each sector image was set to about $40 \mathrm{~Hz}$, which triggered ECG $\mathrm{R}$ wave acquisition. Then, these datasets were stored and transferred to a computer for offline analysis[18, 19]. The 3D datasets are displayed as multiplanar reconstruction images, which correspond to apical 2- and 4-chamber views and three shortaxis levels. And then though the four-dimensional LV analysis software calculated LV end-systolic volume (ESV), LV end-diastolic volume (EDV), LVEF and other parameters. (Figure 1)

\section{D and 3D STE examination}

We used the same ultrasound machine to collect 2D and 3D speckle-tracking images and stored all the datasets digitally in raw data of this machine. The machine was equipped with the commercially available software (4D Auto LVQ software, GE Healthcare) for online quantification of 2D-derived GLS and 3D STE analysis. For 2D strain, apical four-chamber, three-chamber and two-chamber images were simultaneously collected by three-dimensional probe multi-plane mode in the same cardiac cycle. For 3D strain, Image acquisition is the same as real-time 3D full volume imaging. And then we could digitally send the collected data to a separate workstation (EchoPAC PC, 201.1.1) for subsequent offline analysis. The endocardial and epicardial border were traced automatically, and the region of interest was determined by the workstation. The machine automatically obtains 2D-STE and 3D-STE related parameters of the left ventricle[10]. (Figure 2)

If there are any of the following situations in the image, they will be excluded from the statistics: (1) the image quality is poor, and 3 of the 17 segments of the left ventricular wall are rejected. (2) an essential part of the left ventricular wall $(>25 \%)$ is outside the ultrasonic sector. (3) there is a volume rate lower than $40 \%$ of the heart rate in the collected data. (4) the collection contains "suture" artefacts or serious reverb artefacts[20,21].

\section{Statistical analysis.}

Data analysis was performed with SPSS 24.0 software. All results were expressed as means \pm standard deviation (SD). Data from two or more repeated measurements were compared using an ANOVA. The Pearson correlation analysis was used for the analysis of continuous data. All reported p values were twosided, and a $\mathrm{P}<0.05$ was considered an indication of statistical significance.

\section{Results}

In our study, 56 patients were enrolled, and two cases were excluded due to poor image quality. All 54 patients completed anthracycline adjuvant chemotherapy in our hospital. The patients were all women age from 29 to 69 years old, none of the patients had cardiac symptoms, and all patients received dexrazoxane 
during the chemotherapy process. The cumulative dose during chemotherapy was $483.5 \pm 149.0 \mathrm{mg} / \mathrm{m} 2$. Three of the 54 patients began radiotherapy after finished chemotherapy with anthracyclines. The baseline characteristics of the study population are presented in Table 1.

According to the standard 2D echocardiography evaluation: there were no significant changes in the diametral lines of the heart during chemotherapy; the LVEF values and FS values were significantly reduced after six cycles $(\mathrm{P}<0.05)$, although there was a statistically significant reduction in LVEF after anthracycline therapy, none of the patients had heart-related symptoms. In the echo-Doppler parameters, only the E/e' ratio $(\mathrm{P}<0.05)$ was significantly decreased after six cycles, and there was no significant change in left ventricular diastolic function parameters. Of note, representing the overall function of the left ventricle TEI index also was decreased considerably after six cycles (Table 2).

2D STE analyses are shown in Table 2, 3. Compared with the baseline value, left ventricular global longitudinal strain (GLS) were reduced significantly after two, four and six cycles (all $\mathrm{p}<0.05)$. Moreover, the decreasing trend was more obvious with the increase of the dose anthracycline (Table 2). We further discovered that after four cycles the basal anterior segment (BA) and the apical septal segment (AS) were altered significantly (all $\mathrm{P}<0.05$ ); after six cycles the basal anteroseptal segment (BAS) and apex segment were decreased significantly (all $\mathrm{P}<0.05$ ) (Table 2 ).

The 3D volumetric analyses are shown in Table 4. We found that EDV, ESV, and LVEF were reduced significantly after six cycles compared to the baseline values (all $\mathrm{P}<0.05)$, but all patients' LVEF was within the normal range (LVEF $>55 \%$ ).

3D STE analyses are shown in Table 4,5. We found that all 3D strain parameters were significantly decreased after chemotherapy, GLS has reduced dramatically after two cycles, especially. Additionally, compared with baseline values, the basal anterior segment (BA), the apical septal segment (AS) and the Apex segment were significantly reduced after two cycles (all $\mathrm{P}<0.05$ ); the mid-anterior segment $(\mathrm{MA})$, the mid-anteroseptal segment $(\mathrm{BAS})$ and the apical lateral segment $(\mathrm{AL})$ were altered significantly after six cycles $($ all $\mathrm{P}<0.05)$. (Figure 3)

\section{Discussion}

From this study, we tested the diagnostic value of 2D, 3D strain and 3D volumetric analysis in detecting early changes of left ventricular function in breast cancer patients who was undergoing anthracycline chemotherapy. In particular, we further assessed and compared the global and segmental longitudinal strains about the left ventricle derived from $2 \mathrm{D}$ strain and $3 \mathrm{D}$ strain.

The findings of the study indicated that: 1) on standard 2D echocardiographic examination, with the cumulative doses of anthracycline increased 2D derived EF decreased gradually and the E/e' ratio representing left ventricular diastolic function increased significantly; 2) on RT-3DE examination, the 3D volumetric parameters, especially $3 \mathrm{D}$ derived $\mathrm{EF}$ can detect changes that cannot be seen by two-dimensional echocardiography in time; 3) on 2D and 3D STE examination, The subclinical changes of LV longitudinal function can be found in the early stage of the course of the disease, whether it is the GLS parameters derived from 2D strain or the GLS parameters derived from the 3D strain. The difference is that 3D-derived GLS earlier, besides, 3D derived STE all the strain components decreased obviously with the cumulative doses of anthracycline increased. 4) After anthracycline chemotherapy, although the decrease degree of peak systolic strain in each myocardial segment was different, the apical segments were found to be the most vulnerable.

According to the time of occurrence, the myocardial damage caused by anthracyclines can be listed as three categories: acute, chronic, delayed cardiotoxicity. In most patients, cardiotoxicity can occur quickly after anthracycline administration, and the damage is obvious with the extension of time. The chronic and delayed cardiotoxicity of anthracyclines were positively correlated with their cumulative dose[22, 23]. Echocardiography and CMR have the advantage of obtaining information on cardiac structure and function, and both can avoid radiation exposure. More convenient and cheaper echocardiography is the first choice for most patients during monitoring. Monitoring the changes of LVEF is still the basis for the identification 
of cardiotoxicity. From this study, we could find both 2D derived EF, and 3D derived EF decreased with the increased doses of anthracycline[24]. And studies have proved that 3D derived EF and CMR have similar accuracy[25]. However, compared with STE, it is not sensitive to detect early myocardial damage, because a detectable decrease in EF occurs only after a large amount of myocardial tissue is lost, and this is consistent with recent research[26].

Anthracycline-based cardiac toxicity induced by drugs is characterized by myocardial cell death and irreversible cardiac injury. Because the affinity of the anthracycline with myocardial tissue is significantly higher than that of other tissues, so myocardial tissue is more likely to be damaged. The early cardiotoxicity is characterized by cardiomyocyte edema and degeneration, followed by cardiomyocytes myolysis, sarcoplasmic reticulum distortion, replaced by fibrous tissue, and even cardiomyocyte necrosis, resulting in heart failure. The basic structure of myocardium is made up of circumferential fibres in the mid-wall layer, the endocardial layers and epicardial layers are composed of longitudinal and oblique muscle fibres[27]. In an analysis by Crosby et al., researchers found that the absolute values of the peak strain of ventricular septum contraction in each segment after myocardial injury were different, and our results also confirmed this[28]. According to the latest recommended by the American college of cardiology section 17 section method, the left ventricular segmental 17 bull 's-eye map blood supply distribution, the left anterior item first-just branch (left anterior descending, LAD) supply basal anterior(BA), basal anteroseptal(BAS), mid anterior(MA), mid anteroseptal(MAS), apical anterior(AA), apical septal(AS), apex section, right coronary (right coronary artery, RCA) supply basal inferoseptal(BIS), basal lateral(BL), mid inferoseptal(MIS), mid lateral(ML), apical inferior(AI) segment, and the left circumflex branch (left circumflex artery, LCX) supply basal inferolateral(BIL), basal anterolateral(BAL), mid inferolateral(MIL), mid anterolateral(MAL), apical lateral(AL) segment[29]. From the findings of this study, most of the descending segments belong to the blood supply region of the left anterior descending branch, and the trend of the apical segments were the most obvious. And 3D GLS was detected earlier than 2D GLS, which is more meaningful for early detection. 3D-STE the motion of echo spots from three-dimensional space by collecting real-time three-dimensional images of the heart, so it can evaluate the motion of the heart more accurately than 2D-STE.

\section{Limitations}

There are still many limitations to our research. Firstly, the sample size of our study was not large enough, and the follow-up time was not long enough to track the clinical event. But we follow up time was more frequent could detect the early cardiac change in time. Secondly, 3D echocardiography requires a clear and complete endocardial surface, which requires high image quality, and the images we choose were relatively clear and complete. In patients with left mastectomy, the early data cannot be improved one week after the operation due to incision and hematoma, and the late acoustic transparency is worse than that of patients with right mastectomy. Therefore, the number of patients with left mastectomy is small, and the selection of patients' samples was relatively limited.

\section{Conclusion}

In summary, the study showed that using 2D GLS, RT-3DE, and 3D strain to calculate GLS, GCS, GRS, GAS could detect earlier myocardial dysfunction by chemotherapy. And the apical segments of the left ventricle seem to be more susceptible to cardiotoxicity. This provides a new direction for the study of anthracycline myocardial injury, for early myocardial toxicity detection is more accurate to a certain myocardial segment, more targeted timely and effective prevention and treatment.

\section{Acknowledgments}

The authors gratefully acknowledge the financial support by Wenzhou Science and Technology Bureau, Grant No.[Y20190184].

\section{Conflict of Interest}

The authors declare that they have no conflict of interest. 


\section{References}

1.Conway A, McCarthy AL, Lawrence $\mathrm{P}$, et al: The prevention, detection and management of cancer treatment-induced cardiotoxicity: a meta-review. BMC Cancer, 2015. 15 : p. 366.

2.Lenneman CG,Sawyer DB: Cardio-Oncology: An Update on Cardiotoxicity of Cancer-Related Treatment. Circ Res, 2016.118 (6): p. 1008-20.

3.Vasanthakumar A, Kattusamy K,Prasad R: Regulation of daunorubicin biosynthesis in Streptomyces peucetius - feed forward and feedback transcriptional control. J Basic Microbiol, 2013.53 (8): p. 636-44.

4.Narezkina A,Nasim K: Anthracycline Cardiotoxicity. Circ Heart Fail, 2019. 12 (3): p. e005910.

5.Barry E, Alvarez JA, Scully RE, et al: Anthracycline-induced cardiotoxicity: course, pathophysiology, prevention and management.Expert Opin Pharmacother, 2007. 8 (8): p. 1039-58.

6.Edwardson DW, Narendrula R, Chewchuk S, et al: Role of Drug Metabolism in the Cytotoxicity and Clinical Efficacy of Anthracyclines.Curr Drug Metab, 2015. 16 (6): p. 412-26.

7.Zagar TM, Cardinale DM,Marks LB: Breast cancer therapy-associated cardiovascular disease. Nat Rev Clin Oncol, 2016.13 (3): p. 172-84.

8.Zamorano JL, Lancellotti P, Rodriguez Munoz D, et al: 2016 ESC Position Paper on cancer treatments and cardiovascular toxicity developed under the auspices of the ESC Committee for Practice Guidelines: The Task Force for cancer treatments and cardiovascular toxicity of the European Society of Cardiology (ESC). Eur J Heart Fail, 2017. 19 (1): p. 9-42.

9.Santoro C, Arpino G, Esposito R, et al: $2 D$ and $3 D$ strain for detection of subclinical anthracycline cardiotoxicity in breast cancer patients: a balance with feasibility. Eur Heart J Cardiovasc Imaging, 2017. 18 (8): p. 930-936.

10.Mondillo S, Galderisi M, Mele D, et al: Speckle-tracking echocardiography: a new technique for assessing myocardial function. J Ultrasound Med, 2011. 30 (1): p. 71-83.

11.Smiseth OA, Torp H, Opdahl A, et al: Myocardial strain imaging: how useful is it in clinical decision making? Eur Heart J, 2016.37 (15): p. 1196-207.

12.Motoki H, Borowski AG, Shrestha K, et al: Incremental prognostic value of assessing left ventricular myocardial mechanics in patients with chronic systolic heart failure. J Am Coll Cardiol, 2012.60 (20): p. 2074-81.

13.Plana JC, Galderisi M, Barac A, et al: Expert consensus for multimodality imaging evaluation of adult patients during and after cancer therapy: a report from the American Society of Echocardiography and the European Association of Cardiovascular Imaging. Eur Heart J Cardiovasc Imaging, 2014.15 (10): p. 1063-93.

14.Curigliano G, Cardinale D, Suter T, et al: Cardiovascular toxicity induced by chemotherapy, targeted agents and radiotherapy: ESMO Clinical Practice Guidelines. Ann Oncol, 2012. 23 Suppl 7 : p. vii155-66.

15.Negishi K, Negishi T, Hare JL, et al: Independent and incremental value of deformation indices for prediction of trastuzumab-induced cardiotoxicity. J Am Soc Echocardiogr, 2013.26 (5): p. 493-8.

16.Nagueh SF, Appleton CP, Gillebert TC, et al: Recommendations for the evaluation of left ventricular diastolic function by echocardiography. Eur J Echocardiogr, 2009. 10 (2): p. 165-93.

17.Harjai KJ, Scott L, Vivekananthan K, et al: The Tei index: a new prognostic index for patients with symptomatic heart failure. J Am Soc Echocardiogr, 2002. 15 (9): p. 864-8.

18.Lang RM, Bierig M, Devereux RB, et al: Recommendations for chamber quantification: a report from the American Society of Echocardiography's Guidelines and Standards Committee and the Chamber Quantifica- 
tion Writing Group, developed in conjunction with the European Association of Echocardiography, a branch of the European Society of Cardiology. J Am Soc Echocardiogr, 2005. 18 (12): p. 1440-63.

19.Seo Y, Ishizu T, Atsumi A, et al: Three-dimensional speckle tracking echocardiography. Circ J, 2014. 78 (6): p. 1290-301.

20.Galderisi M, Nistri S, Mondillo S, et al: Methodological approach for the assessment of ultrasound reproducibility of cardiac structure and function: a proposal of the study group of Echocardiography of the Italian Society of Cardiology (Ultra Cardia SIC) part I. Cardiovasc Ultrasound, 2011. 9 : p. 26.

21.Yang AQ, Zhang Y, Xu MJ, et al: Use of echocardiography to monitor myocardial damage during anthracycline chemotherapy.Echocardiography-a Journal of Cardiovascular Ultrasound and Allied Techniques, 2019. 36 (3): p. 495-502.

22.Slordal L,Spigset O: Heart failure induced by non-cardiac drugs. Drug Saf, 2006. 29 (7): p. 567-86.

23.Ewer MS,Ewer SM: Long-term cardiac safety of dose-dense anthracycline therapy cannot be predicted from early ejection fraction data. J Clin Oncol, 2009. 27 (36): p. 6073-5.

24.Tarantini L, Cioffi G, Gori S, et al: Trastuzumab adjuvant chemotherapy and cardiotoxicity in real-world women with breast cancer.J Card Fail, 2012. 18 (2): p. 113-9.

25.Hoffmann R, Barletta G, von Bardeleben S, et al: Analysis of left ventricular volumes and function: a multicenter comparison of cardiac magnetic resonance imaging, cine ventriculography, and unenhanced and contrast-enhanced two-dimensional and three-dimensional echocardiography. J Am Soc Echocardiogr, 2014. 27 (3): p. 292-301.

26. Thavendiranathan P,Amir E: Left Ventricular Dysfunction With Trastuzumab Therapy: Is Primary Prevention the Best Option? J Clin Oncol, 2017. 35 (8): p. 820-825.

27.Theodoulou M,Seidman AD: Cardiac effects of adjuvant therapy for early breast cancer. Semin Oncol, 2003. 30 (6): p. 730-9.

28.Crosby J, Amundsen BH, Hergum T, et al: 3-D speckle tracking for assessment of regional left ventricular function. Ultrasound Med Biol, 2009. 35 (3): p. 458-71.

29.Cerqueira MD, Weissman NJ, Dilsizian V, et al: Standardized myocardial segmentation and nomenclature for tomographic imaging of the heart. A statement for healthcare professionals from the Cardiac Imaging Committee of the Council on Clinical Cardiology of the American Heart Association. Circulation, 2002. 105 (4): p. 539-42.

Figure 1 : Real-time 3D full volume data obtained by 4D Automated LV Quantification software(4D Auto LVQ software, GE Healthcare).(A4C: Apical four-chamber; A2C:Apical two-chamber; A3C: Apical threechamber; PSAX: Parasternal short-axis) 


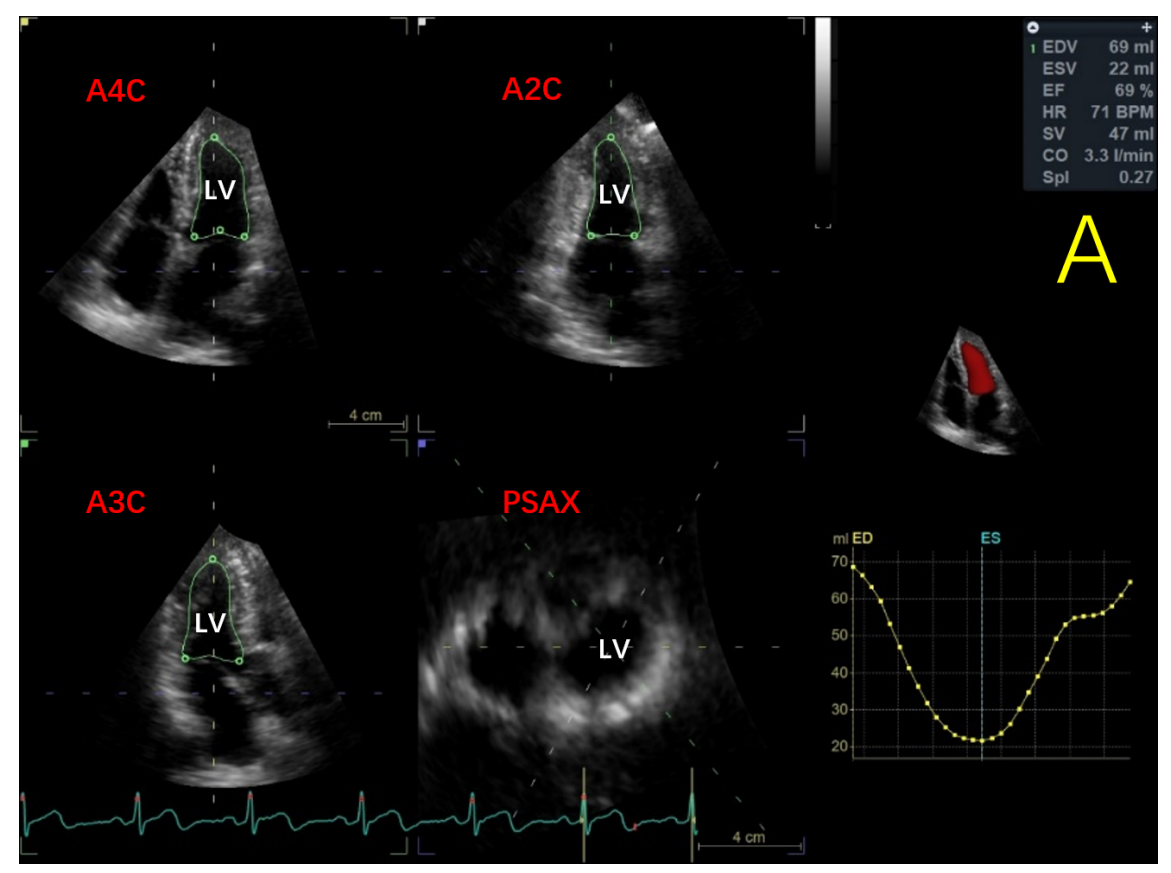

A:The 4D Automated LV Quantification software traced the endocardial.

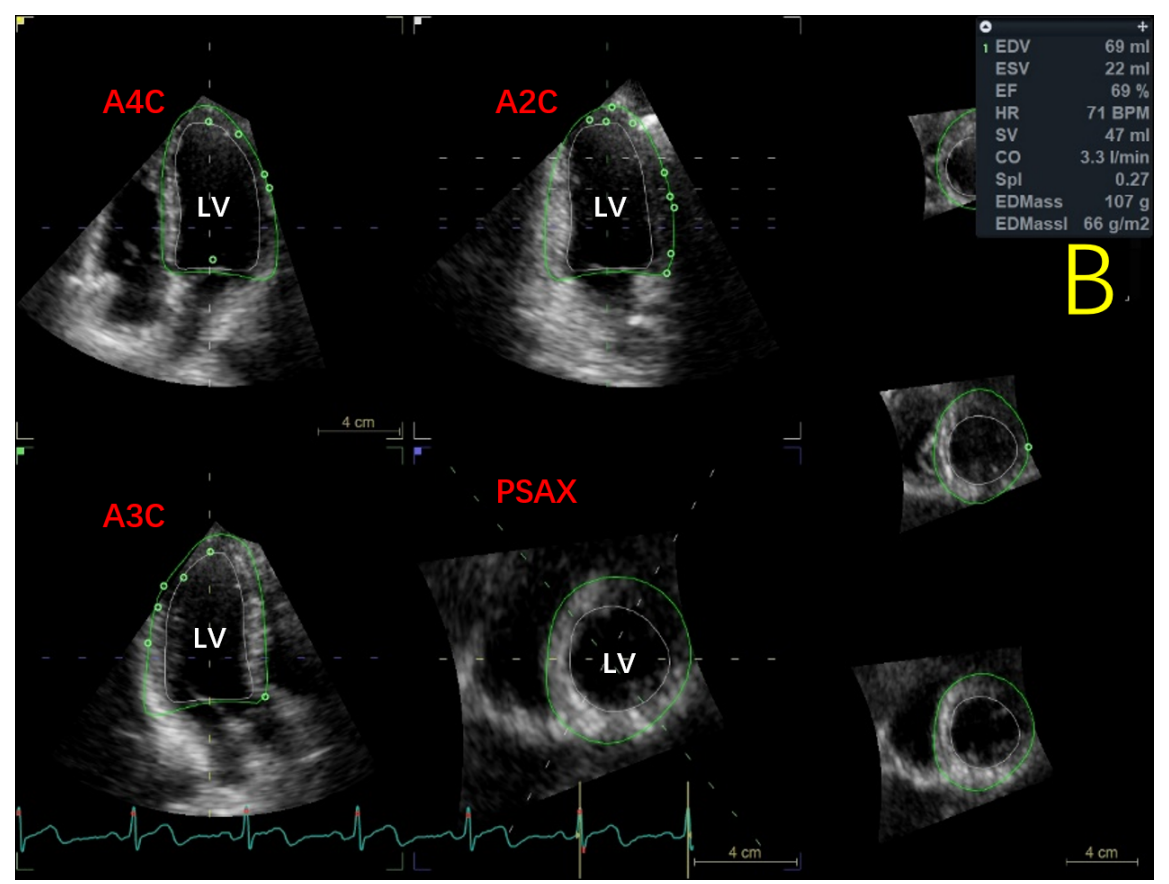

B:The 4D Automated LV Quantification software traced the epicardial border and obtained an entire delineation of the LV wall.

Figure 2 LV's 17 segments bull's eye map and the relationship between the coronary arteries 


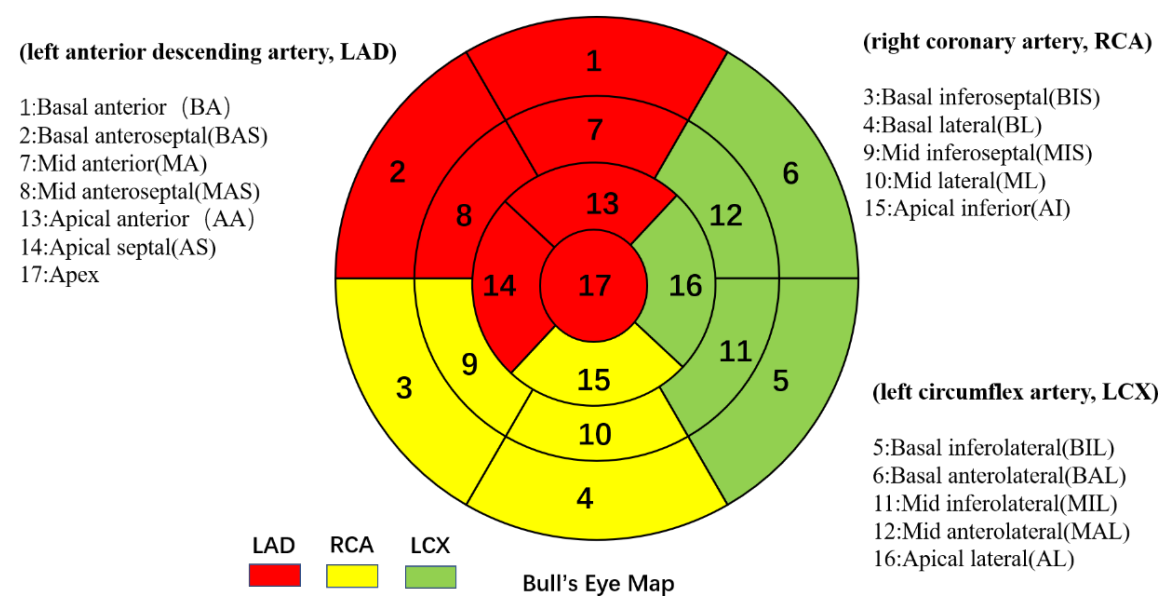

Figure 3: The strain-time curve and 3D STE (bull's eye depiction) in a patient during chemotherapy.
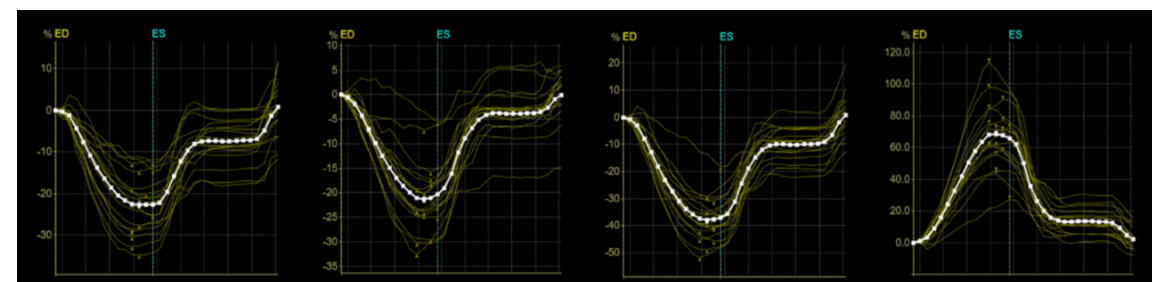

LV-GLS:-23\%

LV-GCS:-21\%

LV-GAS:-38\%

LV-GRS:69\%
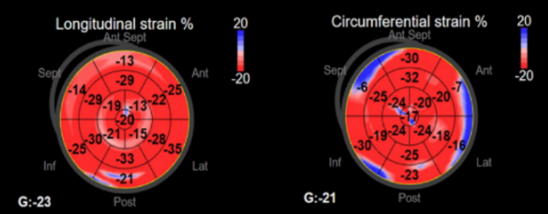

G:21
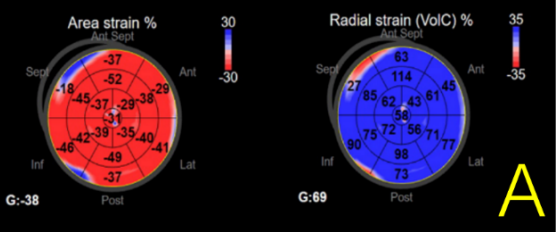

A: The strain-time curve and 3D STE (bull's eye depiction) at baseline(T0).

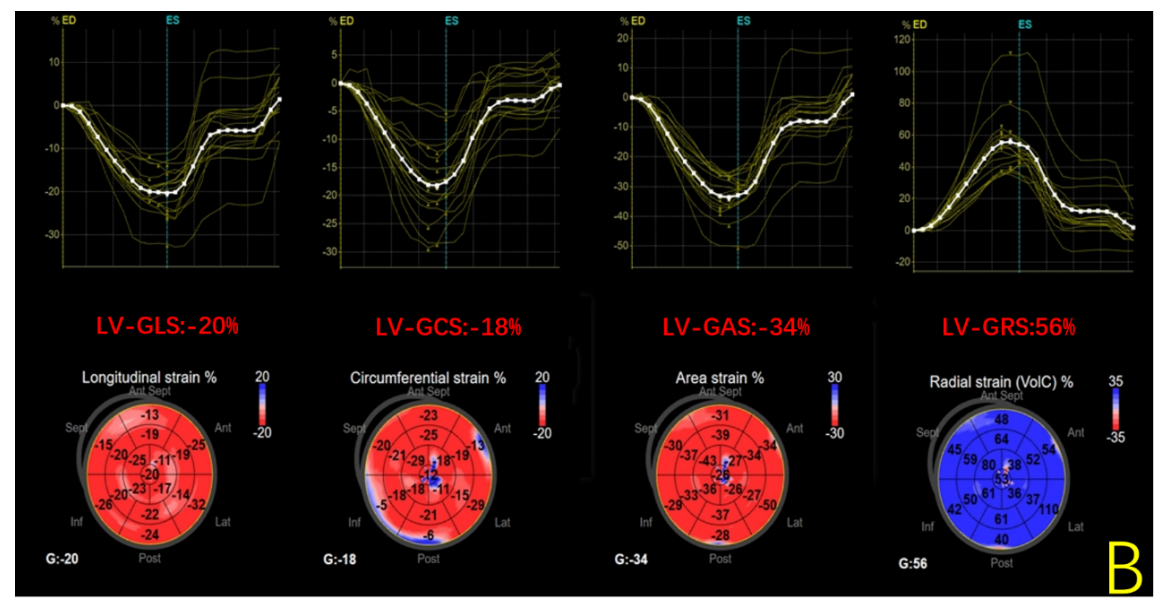


B: The strain-time curve and 3D STE (bull's eye depiction) after two cycles (T2).

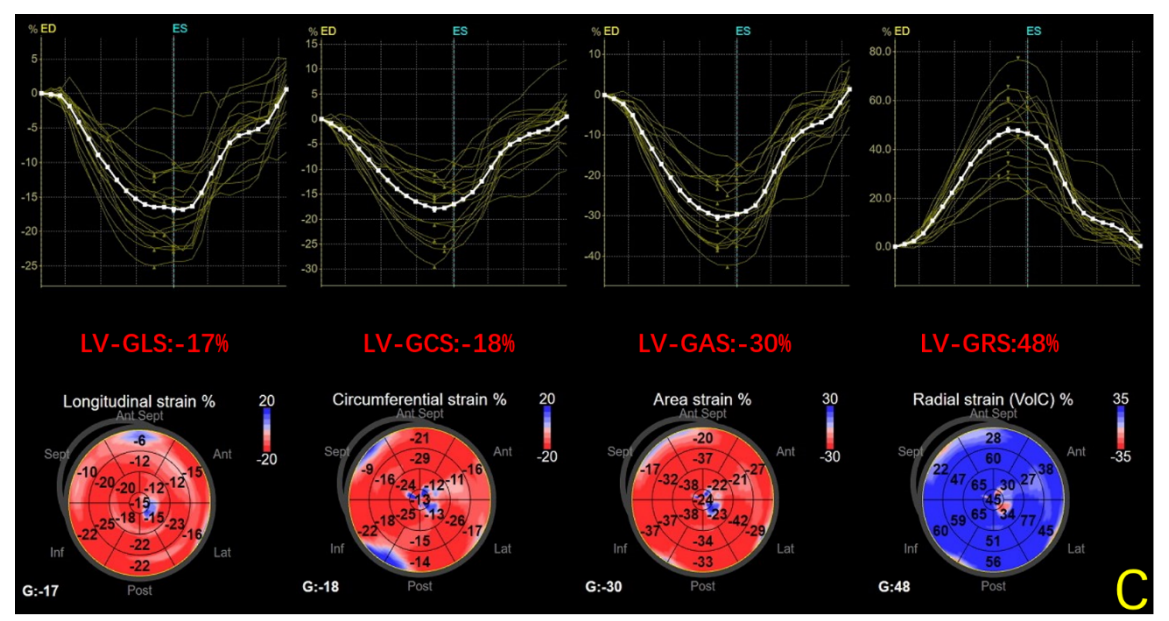

C: The strain-time curve and 3D STE (bull's eye depiction) after six cycles (T6).

Table1 Clinical characteristics of participants at baseline

\begin{tabular}{lll}
\hline Variable & Mean \pm SD & Range \\
\hline Age (years) & $47.6 \pm 8.1$ & $29-68$ \\
Body surface area $(\mathrm{m} 2)$ & $1.6 \pm 0.1$ & $1.4-1.9$ \\
Body mass index $(\mathrm{kg} / \mathrm{m} 2)$ & $24.0 \pm 2.7$ & $20.1-32.1$ \\
Systolic blood pressure $(\mathrm{mmHg})$ & $119.7 \pm 10.8$ & $91-144$ \\
Diastolic blood pressure (mmHg) & $75.9 \pm 8.2$ & $65-96$ \\
Heart rate (bpm) & $78.9 \pm 8.7$ & $60-100$ \\
\hline
\end{tabular}

SD, standard deviation.

Table2 The 2D echocardiographic parameters during chemotherapy.

\begin{tabular}{llllll}
\hline Variable & T0 & T1 & T2 & T4 & T6 \\
\hline LVIDD (mm) & $44.73 \pm 3.20$ & $45.29 \pm 3.21$ & $45.75 \pm 3.13$ & $46.74 \pm 2.99$ & $47.10 \pm 2.95$ \\
LVIDS (mm) & $27.59 \pm 2.35$ & $28.25 \pm 2.28$ & $28.83 \pm 2.26$ & $29.52 \pm 2.28$ & $29.62 \pm 2.14$ \\
LVPW (mm) & $9.23 \pm 0.52$ & $9.32 \pm 0.53$ & $9.46 \pm 0.51$ & $9.66 \pm 0.44$ & $9.77 \pm 0.38$ \\
EDV (mL) & $91.18 \pm 15.64$ & $93.80 \pm 15.23$ & $95.86 \pm 15.35$ & $97.86 \pm 14.63$ & $97.63 \pm 14.47$ \\
ESV (mL) & $28.71 \pm 6.18$ & $30.12 \pm 6.25$ & $31.51 \pm 5.97$ & $32.98 \pm 5.78$ & $33.75 \pm 5.97$ \\
LVM/Ht (g/m2.7) & $37.95 \pm 6.3$ & $38.84 \pm 6.08$ & $39.29 \pm 6.60$ & $40.30 \pm 6.54$ & $41.56 \pm 8.12$ \\
EF (\%) & $68.61 \pm 1.96$ & $67.92 \pm 2.04$ & $65.82 \pm 9.62$ & $65.59 \pm 1.96$ & $63.59 \pm 2.52^{\text {a }}$ \\
FS (\%) & $38.25 \pm 1.25$ & $36.31 \pm 5.42$ & $36.12 \pm 1.73$ & $34.88 \pm 7.31$ & $34.84 \pm 10.87^{\text {a }}$ \\
E/A ratio & $1.19 \pm 0.37$ & $1.19 \pm 0.36$ & $1.20 \pm 0.34$ & $1.19 \pm 0.33$ & $1.23 \pm 0.42$ \\
E velocity DT (ms) & $177.76 \pm 10.09$ & $163.33 \pm 13.24$ & $160.61 \pm 11.03$ & $151.84 \pm 12.17$ & $152.06 \pm 14.50$ \\
E/e' ratio & $9.01 \pm 1.88$ & $9.47 \pm 1.90$ & $9.08 \pm 1.86$ & $9.31 \pm 2.03$ & $9.40 \pm 2.25^{\text {a }}$ \\
GLS & $-21.86 \pm 3.13$ & $-21.46 \pm 2.84$ & $-19.69 \pm 6.477^{\text {a }}$ & $-19.04 \pm 3.00{ }^{\text {a }}$ & $-18.68 \pm 3.27^{\text {a }}$ \\
TEI & $0.35 \pm 0.03$ & $0.35 \pm 0.04$ & $0.36 \pm 0.04$ & $0.38 \pm 0.04$ & $0.42 \pm 0.04^{\text {a }}$ \\
\hline
\end{tabular}


LVIDD, left ventricular internal end-diastolic diameter; LVIDS, left ventricular internal end-systolic diameter; LVPW, left ventricular posterior wall thickness; EDV, end-diastolic volume; ESV, end-systolic volume; $\mathrm{LVM} / \mathrm{Ht}$, left ventricular mass indexed for height; EF, ejection fraction; DT, deceleration time; GLS, global longitudinal strain.

${ }^{\text {a }}$ Compared with T0, $P<0.05$

Table3 The $2 \mathrm{D}$ strain of 17 segments of the left ventricular $(x- \pm s)$

\begin{tabular}{llllll}
\hline & T0 & T1 & T2 & T4 & T6 \\
\hline BA (\%) & $-21.35 \pm 4.50$ & $-19.25 \pm 4.61$ & $-17.98 \pm 4.19$ & $-17.08 \pm 3.75^{\mathrm{a}}$ & $-15.67 \pm 3.91^{\mathrm{a}}$ \\
BAS (\%) & $-17.24 \pm 3.00$ & $-16.37 \pm 3.19$ & $-16.56 \pm 2.92$ & $-15.61 \pm 3.97$ & $-15.32 \pm 3.15^{\mathrm{a}}$ \\
BIS (\%) & $-19.00 \pm 3.09$ & $-17.63 \pm 3.18$ & $-17.92 \pm 3.71$ & $-17.12 \pm 3.29$ & $15.66 \pm 4.75$ \\
BL (\%) & $-17.98 \pm 3.14$ & $-17.65 \pm 3.95$ & $-16.84 \pm 5.54$ & $-16.63 \pm 3.13$ & $14.37 \pm 6.63$ \\
BIL (\%) & $-16.65 \pm 3.99$ & $-17.57 \pm 3.82$ & $-17.02 \pm 3.46$ & $-16.12 \pm 4.45$ & $-16.57 \pm 3.40$ \\
BAL (\%) & $-15.53 \pm 4.92$ & $-15.27 \pm 4.40$ & $-14.58 \pm 3.62$ & $-14.67 \pm 4.12$ & $-12.83 \pm 4.96$ \\
MA (\%) & $-24.96 \pm 5.43$ & $-22.16 \pm 5.89$ & $-21.10 \pm 4.38$ & $-19.78 \pm 4.79$ & $-17.51 \pm 5.05$ \\
MAS (\%) & $-22.18 \pm 3.13$ & $-21.24 \pm 2.75$ & $-20.86 \pm 2.67$ & $-19.98 \pm 2.95$ & $-18.88 \pm 2.97$ \\
MIS (\%) & $-21.94 \pm 3.97$ & $-21.31 \pm 2.80$ & $-20.10 \pm 7.03$ & $-19.63 \pm 3.33$ & $-17.90 \pm 4.41$ \\
ML (\%) & $-21.41 \pm 3.85$ & $-20.29 \pm 3.86$ & $-19.98 \pm 4.34$ & $-18.34 \pm 3.41$ & $-16.80 \pm 4.13$ \\
MIL (\%) & $-19.98 \pm 4.07$ & $-20.12 \pm 3.49$ & $-18.78 \pm 3.68$ & $-17.10 \pm 4.45$ & $-17.04 \pm 3.36$ \\
MAL (\%) & $-20.57 \pm 4.10$ & $-19.06 \pm 5.45$ & $-18.10 \pm 5.21$ & $-17.24 \pm 3.92$ & $-15.00 \pm 7.19$ \\
AA (\%) & $-28.78 \pm 4.74$ & $-26.78 \pm 4.53$ & $-24.64 \pm 4.5$ & $-23.57 \pm 4.95$ & $-21.57 \pm 4.60$ \\
AS (\%) & $-28.57 \pm 4.94$ & $-26.78 \pm 4.62$ & $-25.20 \pm 4.37$ & $-23.00 \pm 4.95$ & $-20.63 \pm 5.60$ a \\
AI (\%) & $-26.96 \pm 4.82$ & $-25.37 \pm 4.09$ & $-23.00 \pm 5.03$ & -21.29 .6 .96 & $-19.61 \pm 5.13$ \\
AL (\%) & $-28.69 \pm 5.56$ & $-26.08 \pm 6.04$ & $-23.98 \pm 6.5$ & $-21.86 \pm 5.37$ & $-18.59 \pm 6.01$ \\
Apex (\%) & $-28.31 \pm 4.50$ & $-26.22 \pm 4.48$ & $-22.63 \pm 4.40$ & $-22.63 \pm 4.40$ & $-20.30 \pm 4.49^{\text {a }}$ \\
\hline
\end{tabular}

a Compared with T0, $P<0.05$

Table4 The 3D echocardiographic parameters during chemotherapy.

\begin{tabular}{llllll}
\hline Variable & T0 & T1 & T2 & T4 & T6 \\
\hline EDV (mL) & $80.33 \pm 15.54$ & $84.78 \pm 15.03$ & $87.63 \pm 14.73$ & $89.20 \pm 14.39$ & $91.84 \pm 14.11^{\text {a }}$ \\
ESV (mL) & $31.12 \pm 7.09$ & $33.76 \pm 7.65$ & $35.33 \pm 7.55$ & $36.90 \pm 7.09$ & $38.98 \pm 7.59$ a $^{\circ}$ \\
SV (mL) & $49.71 \pm 9.36$ & $51.25 \pm 9.32$ & $52.55 \pm 7.92$ & $52.73 \pm 8.67$ & $54.31 \pm 8.29$ \\
CO (L/m) & $3.68 \pm 0.76$ & $3.89 \pm 0.88$ & $3.95 \pm 0.79$ & $4.01 \pm 8.67$ & $4.17 \pm 0.74$ \\
EF (\%) & $62.18 \pm 2.86$ & $60.84 \pm 3.34$ & $60.00 \pm 3.11$ & $59.16 \pm 3.09$ & $58.53 \pm 4.15^{\text {a }}$ \\
SpI & $0.33 \pm 0.07$ & $0.34 \pm 0.07$ & $0.34 \pm 0.08$ & $0.34 \pm 0.79$ & $0.36 \pm 0.07$ \\
LVM/Ht (g/m2.7) & $41.82 \pm 5.23$ & $42.68 \pm 4.57$ & $43.71 \pm 4.43$ & $43.87 \pm 5.40$ & $44.71 \pm 5.52$ \\
GLS (\%) & $-19.02 \pm 3.25$ & $-17.84 \pm 3.18$ & $-16.51 \pm 3.06$ & $-15.37 \pm 2.83^{a}$ & $-14.02 \pm 4.19^{\text {a }}$ \\
GCS (\%) & $-17.76 \pm 3.55$ & $-15.84 \pm 3.47$ & $-15.20 \pm 3.14$ & $-13.47 \pm 3.18{ }^{a}$ & $-10.92 \pm 8.55^{\text {a }}$ \\
GAS (\%) & $-31.18 \pm 5.03$ & $-28.82 \pm 4.94$ & $-27.06 \pm 4.31$ & $-25.39 \pm 4.42$ & $-22.55 \pm 5.93^{\text {a }}$ \\
GRS (\%) & $53.24 \pm 12.90$ & $46.98 \pm 10.53$ & $43.90 \pm 10.23$ & $39.02 \pm 13.45$ & $35.90 \pm 8.52^{\text {a }}$ \\
\hline
\end{tabular}

SV, stroke volume; CO, cardiac output; GCS, global circumferential strain; GAS, global area strain; GRS, global radial strain; SpI, Sphericity Index

${ }^{\text {a }}$ Compared with T0, $P<0.05$

Table 5 The $3 \mathrm{D}$ strain of 17 segments of the left ventricle $(\mathrm{x}- \pm \mathrm{s})$ 


\begin{tabular}{llllll}
\hline & T0 & T1 & T2 & T4 & T6 \\
\hline BA (\%) & $-16.75 \pm 6.10$ & $-14.75 \pm 7.17$ & $-13.96 \pm 5.54^{\mathrm{a}}$ & $-12.24 \pm 8.04^{\mathrm{a}}$ & $-11.60 \pm 7.55^{\mathrm{a}}$ \\
BAS (\%) & $-17.86 \pm 5.36$ & $-16.65 \pm 4.08$ & $-15.69 \pm 6.20$ & $-15.73 \pm 4.83$ & $-15.25 \pm 5.12$ \\
BIS (\%) & $-26.84 \pm 6.42$ & $-24.76 \pm 6.56$ & $-22.86 \pm 6.90$ & $-22.06 \pm 5.90$ & $-19.93 \pm 8.29$ \\
BL (\%) & $-23.59 \pm 9.51$ & $-23.51 \pm 8.43$ & $-21.92 \pm 7.42$ & $-19.50 \pm 10.28$ & $-15.00 \pm 10.48$ \\
BIL (\%) & $-29.67 \pm 9.02$ & $-26.76 \pm 9.74$ & $-24.39 \pm 11.14$ & $-21.94 \pm 11.39$ & $-19.17 \pm 11.57$ \\
BAL (\%) & $-25.63 \pm 8.44$ & $-23.10 \pm 8.67$ & $-22.17 \pm 8.64$ & $-20.64 \pm 7.19$ & $-18.88 \pm 7.84$ \\
MA (\%) & $-16.84 \pm 6.27$ & $-15.86 \pm 6.19$ & $-13.63 \pm 7.36$ & $-13.42 \pm 5.75$ & $-13.35 \pm 6.16^{\text {a }}$ \\
MAS (\%) & $-20.43 \pm 3.47$ & $-19.31 \pm 3.55$ & $-18.63 \pm 4.23$ & $-17.94 \pm 3.81$ & $-17.29 \pm 3.57^{\text {a }}$ \\
MIS (\%) & $-22.37 \pm 3.91$ & $-20.57 \pm 4.02$ & $-20.85 \pm 4.31$ & $-20.19 \pm 4.18$ & $-17.85 \pm 5.68$ \\
ML (\%) & $-16.86 \pm 8.23$ & $-14.74 \pm 8.43$ & $-14.45 \pm 8.73$ & $-13.52 \pm 8.85$ & $-11.02 \pm 9.89$ \\
MIL (\%) & $-13.90 \pm 9.28$ & $-12.88 \pm 8.37$ & $-11.30 \pm 8.71$ & $-9.95 \pm 8.98$ & $-6.06 \pm 8.90$ \\
MAL (\%) & $-14.73 \pm 8.00$ & $-11.86 \pm 9.05$ & $-10.16 \pm 7.87$ & $-10.44 \pm 7.88$ & $-8.60 \pm 7.60$ \\
AA (\%) & $-18.58 \pm 4.91$ & $-18.63 \pm 4.16$ & $-16.10 \pm 6.88$ & $-17.06 \pm 4.63$ & $-17.22 \pm 4.74$ \\
AS (\%) & $-19.37 \pm 5.79$ & $-17.06 \pm 8.86$ & $-17.59 \pm 5.13^{a}$ & $-16.60 \pm 5.02$ & $-14.50 \pm 5.95$ \\
AI (\%) & $-13.16 \pm 6.76$ & $-12.45 \pm 6.78$ & $-11.59 \pm 6.90$ & $-10.24 \pm 7.99$ & $-8.42 \pm 6.98$ \\
AL (\%) & $-13.61 \pm 5.13$ & $-12.47 \pm 6.68$ & $-12.25 \pm 4.20$ & $-11.51 \pm 4.04$ & $-11.08 \pm 5.38^{\text {a }}$ \\
Apex (\%) & $-11.94 \pm 6.64$ & $-10.76 \pm 6.23$ & $-11.94 \pm 6.64{ }^{a}$ & $-9.00 \pm 6.79$ a & $-8.52 \pm 8.02^{\text {a }}$ \\
\hline
\end{tabular}

${ }^{\text {a }}$ Compared with T0, $P<0.05$

\section{Hosted file}

Figure\#1A.pdf available at https://authorea.com/users/370852/articles/489365-using-2dand-3d-strain-to-detect-myocardial-damage-during-anthracycline-chemotherapy-in-breastcancer-patients

\section{Hosted file}

Figure\#1B.pdf available at https://authorea.com/users/370852/articles/489365-using-2dand-3d-strain-to-detect-myocardial-damage-during-anthracycline-chemotherapy-in-breastcancer-patients

\section{Hosted file}

Figure\#2.pdf available at https://authorea.com/users/370852/articles/489365-using-2d-and-3dstrain-to-detect-myocardial-damage-during-anthracycline-chemotherapy-in-breast-cancerpatients

\section{Hosted file}

Figure\#3A.pdf available at https://authorea.com/users/370852/articles/489365-using-2dand-3d-strain-to-detect-myocardial-damage-during-anthracycline-chemotherapy-in-breastcancer-patients

\section{Hosted file}

Figure\#3B.pdf available at https://authorea.com/users/370852/articles/489365-using-2dand-3d-strain-to-detect-myocardial-damage-during-anthracycline-chemotherapy-in-breastcancer-patients

\section{Hosted file}

Figure\#3C.pdf available at https://authorea.com/users/370852/articles/489365-using-2dand-3d-strain-to-detect-myocardial-damage-during-anthracycline-chemotherapy-in-breastcancer-patients 